\title{
Complex Assessment of Risk Factors for the Development of Cardiovascular Calcification in Hemodialysis Patients
}

\author{
Liliya I. Rudenko, PhD*; Mikhail M. Batiushin, $\mathrm{PhD}, \mathrm{ScD}$; \\ Aleksandr A. Kastanayan, PhD, ScD; Dmitriy G. Pasechnik, PhD, ScD; \\ Igor V. Dudarev; Elena A. Kartashova, PhD; Ekaterina S. Lapina \\ Rostov State Medical University \\ Rostov-on-Don, the Russian Federation
}

\begin{abstract}
The aim of the present study was the integrated assessment of the role of non-traditional factors (inflammation, malnutrition, calcium-phosphorus disorder and imbalance in the concentration of inducers and inhibitors of calcification) in forming cardiovascular calcification (CVC) and the structural-functional rearrangement of LV myocardium in patients with chronic kidney disease (CKD) receiving hemodialysis (HD).

Materials and Methods: The present study included $84 \mathrm{HD}$ patients with CKD 5D stage. We evaluated 3 components of the Dialysis Malnutrition Score (DMS), according to which body mass index (BMI), the level of serum albumin, and the percent saturation of transferrin with iron were determined. We also analyzed CRP, fibrinogen, and beta-2 microglobulin, and calculated the number of points (from zero to 2) according to the Glasgow Prognostic Score (GPS), which allowed us to combine indicators of inflammation and make a common prognostic assessment.

The serum levels of protein alpha-Klotho и FGF-23 were determined by enzyme immunoassay. Echocardiographic measurements were performed using B-mode, M-mode and Doppler-mode. Different patterns of LV geometry were identified according to Ganau et al. (1992). The severity of calcification was estimated by a semi-quantitative scale for assessing the degree of calcification of heart structures according to the National recommendations for CKD-MBD (2010).

Results: The increased risk for development of CVC, LVH, and diastolic dysfunction was associated with markers of malnutrition, anemia, and inflammation in HD patients. Reduced serum alpha-Klotho level, hypoalbuminemia and a high level of FGF-23 had a prognostic value in CVC formation. (Int J Biomed. 2016;6(1):22-26.).
\end{abstract}

Keywords: chronic kidney disease; end-stage renal disease; renal replacement therapy; cardiovascular calcification.

\section{Abbreviations}

CVD, cardiovascular disease; CKD, chronic kidney disease; LVH, left ventricular hypertrophy; IVST, interventricular septal thickness; LVPWT, left ventricular posterior wall thickness; LVMI, left ventricular mass index; CRP, C-reactive protein.

\section{Introduction}

In recent years, the number of patients with end-stage renal disease (ESRD) who require renal replacement therapy (RRT) is increasing dramatically worldwide (KDIGO, 2012). The main cause of mortality among dialysis patients with ESRD is CVD, which occurs more often in patients receiving

*Corresponding author: Liliya I. Rudenko, PhD. Rostov State Medical University, Rostov-on-Don, Russia. E-mail: rudenko. liliya@mail.ru hemodialysis (HD), than in the general population [1]. The frequency of deaths from CVD is high among young dialysis patients, despite the fact that in the population cardiovascular mortality progresses with age [2]. Thus, ESRD has not only medical but also socio-economic significance, and thus requires researching and clarifying mechanisms of calcification to find new diagnostic methods and make predictive algorithms of CVC development in dialysis patients.

The increased risk of adverse CVD outcomes is largely due to calcification of heart valves and vessel walls, which is an element of CKD- mineral bone disorder (CKD-MBD) along 
with the imbalance in the metabolism of calcium, phosphorus, parathormone (PTH) and bone tissue. Previously, the calciumphosphorus imbalance was estimated as renal osteodystrophy that was characterized by morpho-functional disorders of bone tissue, and then it was found that laboratory abnormalities in the metabolism of calcium, phosphorus and PTH levels are independent indicators of prognosis and are associated with the progression of $\mathrm{CKD}$, cardiovascular complications and mortality $[3,4]$.

Understanding the risk factors, etiology and pathogenesis of CKD-MBD has significantly improved due to data on known markers of mineral metabolism, and researchers have identified previously unknown factors that are components of renal failure. In addition to the previously known participants of the calcium-phosphorus homeostasis, new elements of metabolism are being studied, elements that could be involved in the process of CVC. In CKD patients, calcification is associated with both traditional risk factors, such as age, diabetes, hypertension, smoking, dyslipidemia, and non-traditional factors, including decreased GFR, anemia, hyperphosphatemia, secondary hyperparathyroidism (sHPT), inflammation, oxidative stress and imbalance in the concentration of inducers and inhibitors of calcification.

The aim of the present study was the integrated assessment of the role of non-traditional factors in forming $\mathrm{CVC}$ and the structural-functional rearrangement of LV myocardium in CKD patients receiving hemodialysis (HD).

\section{Materials and Methods}

The present study included $84 \mathrm{HD}$ patients with CKD 5D stage (45 male and 39 female; mean age $53.5 \pm 14.9$ years). The study was conducted in accordance with ethical principles of the Declaration of Helsinki and approved by the Rostov State Medical University Ethics Committee. Written informed consent was obtained from all participants.

All HD patients received online hemodiafiltration treatments three times per week for 4-5h, with ultra-pure bicarbonate-based dialysate using a high flux polysulfone membrane.

Exclusion criteria were age $<18$ or $>80$ years, pathology of the parathyroid glands, uncontrolled intake of vitamin D and/or calcimimetics, the history for drug and alcoholism, pathology of the bone tissue, mental disorders, kidney transplant.

We evaluated 3 components of the Dialysis Malnutrition Score (DMS), according to which body mass index (BMI), the level of serum albumin, and the percent saturation of transferrin with iron were determined. We also analyzed CRP, fibrinogen, and beta- 2 microglobulin, and calculated the number of points (from zero to 2) according to the Glasgow Prognostic Score (GPS), which allowed us to combine indicators of inflammation and make a common prognostic assessment [5].

The serum levels of protein alpha-Klotho и FGF-23 were determined by enzyme immunoassay. The average level of serum FGF-23 was $69.3 \pm 23.5 \mathrm{pg} / \mathrm{ml}$, alpha-Klotho $460.4 \pm 141.3 \mathrm{pg} / \mathrm{ml}$.
Serum levels of calcium, inorganic phosphorus, calciumphosphorus compositions, intact PTH (iPTH) were investigated. sHPT was defined as PTH level $>300 \mathrm{pg} / \mathrm{ml}$; hyperphosphatemia at the phosphate concentration $>1.45 \mathrm{mmol} / 1$ (the National recommendations for CKD-MBD, 2010).

Echocardiographic measurements were performed using B-mode, M-mode and Doppler-mode. Different patterns of LV geometry were identified according to Ganau et al. (1992).

The severity of calcification was estimated by a semiquantitative scale for assessing the degree of calcification of heart structures according to the National recommendations for CKD-MBD (2010). Patients underwent plain radiography of the abdominal cavity in lateral projection for the assessment of abdominal aortic calcification (AAC). Calcification was assessed using Kauppila's scale, which allowed us to assess the severity of calcification in the aorta at the level of the first through fourth lumbar vertebrae, and in the anterior and posterior walls of the aorta. Calcific densities were graded on a 0 to 3 scale at each lumbar vertebral segment. A score of 0 denoted no aortic calcific deposits; 1 , small scattered calcific deposits filled less than one-third of the longitudinal wall of the aorta; 2, one-third or more but less than two-thirds of the longitudinal wall of the aorta was calcified; and 3, two-thirds or more of the longitudinal wall of the aorta was calcified. A separate score was determined for the anterior and posterior aorta, and the values were summed across the 4 vertebrae, resulting in an AAC index that could range from 0 to 24 points [6].

Statistical analysis of data was performed using the software Statistica 6.0. The mean (M) and standard deviation (SD) were calculated. For data with normal distribution, inter-group comparisons were performed using Student's t-test. Comparisons between three groups were performed with the one-way ANOVA with Tukey's post-hoc test. Group comparisons with respect to categorical variables are performed using $\chi^{2}$ tests. Linear and non-linear regression analysis, as well as logistic regression analysis was conducted. A probability value of $\mathrm{P}<0.05$ was considered statistically significant.

\section{Results}

CVC was revealed in $42 / 50 \%$ of HD patients. Increased risk of calcification was detected in patients with low total protein $(P=0.003)$. A decrease in the level of total protein, even by $2 \mathrm{~g} / \mathrm{l}$, increased the risk of calcification by more than $5 \%$. Low albumin levels significantly increased CVC risk $(P=0.01)$. The average albumin level in patients with calcification was detected in the lower range $(37.2 \pm 4.4 \mathrm{~g} / \mathrm{l})$ compared with patients without diagnosed calcification $(39.7 \pm 3.9 \mathrm{~g} / \mathrm{l}, \mathrm{p}<0.01)$; this can be explained by the role of malnutrition in the development of calcification. Thus, malnutrition, specifically low levels of albumin and total protein, is associated with a rising risk of CVC.

Patients with calcification of heart valves had a higher pulse pressure compared with patients without calcification before ( $66.2 \pm 16.9$ vs $58.5 \pm 12.3$, respectively, $P=0.02)$, during $(65.1 \pm 14.4$ vs $57.3 \pm 13.0$, respectively, $P=0.01)$ and after hemodialysis ( $62.9 \pm 13.3$ vs $56.6 \pm 14.3$, respectively, $P<0.05$ ) 
and in the interdialytic period $(63.4 \pm 19.6$ vs $51.5 \pm 14.4$, respectively, $P<0.01$ ).

The echocardiography performed in the interdialytic period showed that the increasing degree of calcification in HD patients increased the risk of LVH $(P=0.03)$, likely due to the hemodynamic changes because of existing areas of calcification. According to the degree of calcification of cardiac structures by a semi-quantitative scale, a 2-point score leads to an increase in the probability of LVH up to $71.9 \%$, and in cases of maximum severity, up to $91.9 \%$. We identified a positive correlation between the severity of heart valve calcification and LVMI (an index of myocardial mass) ( $\mathrm{r}=0.27, P=0.016$ ). Among patients with diagnosed CVC, LVMI was higher than in patients without changes in the valves $(132.8 \pm 31.4$ and $114.4 \pm 34.9 \mathrm{~g} / \mathrm{m}^{2}$, respectively, $P=0.02$ ). Characteristics are presented in the Table 1. Furthermore, IVST and LVPWT had a positive correlation with the severity of calcification ( $\mathrm{r}=0.329, P=0.01$ and $\mathrm{r}=0.31, P=0.02$, respectively).

\section{Table 1.}

Characteristics of clinical status of HD patients with or without CVC

\begin{tabular}{|l|c|c|c|}
\hline \multicolumn{1}{|c|}{ Criterion } & $\begin{array}{c}\text { Patients with } \\
\text { CVC- } \\
(\mathrm{n}=42)\end{array}$ & $\begin{array}{c}\text { Patients with } \\
\text { CVC+ } \\
(\mathrm{n}=42)\end{array}$ & $p$ \\
\hline Serum protein, $\mathrm{g} / \mathrm{l}$ & $71.05 \pm 4.26$ & $67.3 \pm 4.49$ & $<0.01$ \\
\hline Serum albumin, $\mathrm{g} / \mathrm{l}$ & $39.7 \pm 3.9$ & $37.2 \pm 4.4$ & $<0.01$ \\
\hline PP (before HD), $\mathrm{mmHg}$ & $58.5 \pm 12.3$ & $66.2 \pm 16.9$ & $<0.02$ \\
\hline PP (during HD), $\mathrm{mmHg}$ & $57.3 \pm 13.0$ & $65.1 \pm 14.4$ & $=0.01$ \\
\hline PP (after HD), $\mathrm{mmHg}$ & $56.6 \pm 14.3$ & $62.9 \pm 13.3$ & $<0.04$ \\
\hline PP (interdialytic period), $\mathrm{mmHg}$ & $51.5 \pm 14.4$ & $63.4 \pm 19.6$ & $<0.01$ \\
\hline LVMI, g/m ${ }^{2}$ & $114.4 \pm 34.9$ & $132.8 \pm 31.4$ & $<0.02$ \\
\hline
\end{tabular}

$P P$ - pulse pressure

LV remodeling was identified in 63/76.8\% of HD patients. Concentric remodeling, concentric hypertrophy, and eccentric LVH were detected in $31 / 37.8 \%, 26 / 31.7 \%$ and $9 / 7.3 \%$ of patients, respectively. The diastolic dysfunction was identified in $70.4 \%$ of HD patients. An impaired relaxation pattern or grade 1 diastolic dysfunction was detected in $53 / 65,4 \%$ of patients and pseudonormal mitral inflow pattern or grade 2 diastolic dysfunction in $4 / 5,0 \%$ of patients. Restrictive filling dynamics (grade 3 and 4 DD) were not identified among the studied patients.

In the presence of cardiovascular calcification, the average DMS score was significantly higher $(1.07 \pm 0.62$ points), whereas in patients without calcification it was $0.40 \pm 0.57$ points $(P<0.01)$. One of the DMS components in HD patients is albumin; we found a significant negative correlation between the albumin level and LVMI ( $\mathrm{r}=-0.32$, $P=0.003)$.

Parameters of systemic inflammation were associated with LVH; the increasing GPS score significantly increased the manifestation of LVH and an increase in left atrium size. The presence of high phosphorus and two GPS points in HD patients increased the risk of CVC up to $92.2 \%$, whereas in patients with normal phosphorus and zero GPS points, the risk was only $11.5 \%(P<0.05)$. In CKD patients, CRP is a marker of an inflammatory response. However, the role of CRP as a predictor of the development of CVC is ambiguous.

Logistic analysis showed that the increased CRP levels significantly increased the risk of CVC $(P=0.04)$.

Patients with anemia had a larger LVPWT in comparison with patients with a normal range of hemoglobin $(P=0.01)$. In patients with diagnosed $\mathrm{CVC}$, hemoglobin level was lower than in the patients without calcification $(P<0.01)$. Thus, the risk of CVC in patients with latent anemia amounted to $15.5 \%$. In patients with the first degree of anemia, the probability of calcification was in the range of $15.5 \%$ to $36.6 \%$; with the second degree, $37 \%$ to $64 \%$; and with the third degree, more than $64.4 \%(P=0.03)$.

An increase in serum FGF-23 level and a decrease in serum alpha-Klotho level were identified in patients with progression of renal dysfunction. A negative correlation was established between the duration of dialysis and the alphaKlotho level ( $\mathrm{r}=-0.325, P=0.006)$. When the alpha-Klotho concentration was about $800 \mathrm{pg} / \mathrm{ml}, \mathrm{CVC}$ risk was minimal and amounted only 4\%; a reduction in the protein level by $50 \%$ increased the risk almost 6 times up to $23.3 \%$, and a fourfold decrease, up to $43.3 \%$. While the level of alpha-Klotho was below $100 \mathrm{pg} / \mathrm{ml}$, the probability of the aortic calcification was more than $50 \%(P=0.02)$. The gain of $\mathrm{CVC}$ risk also was found in patients with high serum FGF-23 level and persistent high rates of urea $(P=0.048)$. The $\mathrm{CVC}$ risk increased in patients with hypoalbuminemia and rising levels of FGF-23 $(P=0.01)$. Thus, hypoalbuminemia with a high level of FGF23 had a prognostic value in $\mathrm{CVC}$ formation.

\section{Discussion}

Given that CKD is considered a disease, manifested not only as a mineral bone disorder, components of the system's inflammatory response, malnutrition and anemia were analyzed to confirm the high risk of calcification in studied patients. It is known that protein-energy malnutrition is a predictor of mortality in patients with ESRD [7]. One of the most common markers of malnutrition is hypoalbuminemia [8]; low levels of this protein are involved in impeding the transport of calcium, phosphorus and other electrolytes, which can contribute to arterial calcification. We found that low serum albumin correlated with the severity of calcification. In addition, there was a negative correlation between the albumin level and the relationship between early diastolic mitral annular velocity (EA) and tissue Doppler diastolic velocity (Ea), which partially describes the participation of malnutrition in the development of DD in HD patients.

Inflammation is one of the fundamental pathophysiological phenomena associated with renal failure, particularly among patients receiving chronic $\operatorname{HD}[9,10]$. L. Yan et al., in models on mice, found that an enhanced production of inflammatory cytokines induces the development of myocardial hypertrophy [11]. In addition, a few years earlier S.Barry described the role of proinflammatory chemokines in LVH formation [12].

In most patients (64.3\%), we registered an increase in 
CRP level and found that the higher CRP levels increased the probability of CVC. In addition, the likelihood of DD was increased with the growth of CRP level.

In the study [13] examining inflammation and LV diastolic dysfunction in peritoneal dialysis (PD) patients, the role of pro-inflammatorycytokines in a group of PD patients and controls was assessed. That study identified a significant correlation between LV diastolic dysfunction and serum TNF-a, and IL-6 levels in PD patients and an interaction between PD and inflammation. TNF-a was also shown to aggravate LV diastolic dysfunction.

Analyzing the parameters according to GPS, we found that GPS points in patients with CVC were higher than among people without calcification. The likelihood of developing calcification reached $60 \%$ in patients with maximum points. In addition, high GPS points increased the risk of LVH development, which is positively correlated with LVMI and LVPWT. Evidence is accumulating that chronic inflammation plays an important role in LVH. Cytokines are implicated in the development of cardiac hypertrophy, most likely via downstream activation of pro-inflammatory transcription

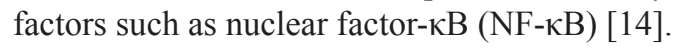

The participation of albumin in LVH development is not completely known; however, there is an assumption that malnutrition in combination with chronic overload in dialysis patients contributes to an increase in the extracellular fluid volume, which is a risk factor for LVH development..

Persistent uremia and constant contact with the dialysis membrane, the release of pro-inflammatory cytokines, oxidative stress, and persistent activation of the inflammatory response [15] underlie the changes in morphological and functional properties of smooth muscle cells and development of calcification. The role of another acute-phase protein, $\beta 2-$ microglobulin, was statistically significant only in patients with an elevated pulse pressure estimated in the interdialytic period. Thus, it was found that the enlargement in pulse pressure and an increased level of $\beta 2$-microglobulin contribute to the increasing probability of CVC development due to progressive vascular stiffness in CKD patients. According to M.Masuda et al. [16], serum $\beta 2-\mathrm{MG}$ concentrations correlated significantly and positively with the echocardiographic parameters of left ventricular hypertrophy (LVH) in long-term HD patients; a deposition of $\beta 2-\mathrm{MG}$ amyloid in the heart may be associated with LVH progression.

Thus, the obtained data on the relationship of systemic inflammation, cardiovascular calcification and anatomical and functional changes in the heart in patients receiving chronic HD, suggest that continuous activation of inflammatory responses in this category of patients is an important independent risk factor for cardiovascular disorders in dialysis patients.

Our data revealed that the ferritin level positively correlated with the degree of valve calcification, EA, and Ea in HD patients. In addition, the relationship between the fibrinogen level and LVM can be explained by the increased load on the myocardium under hyperfibrinogenemia.

Anemia contributes to the development of hemodynamic and non-hemodynamic mechanisms, which results in a compensatory increase in hemoglobin levels. Hemodynamic compensatory changes in existing chronic anemia include an increasing cardiac output because of reduced blood viscosity and vasodilation contributing to tissue perfusion. Anemia leads to stimulation of the sympathetic nervous system as well as to activation of the renin-angiotensin-aldosterone system, which is closely associated with increased oxidative stress [17] and defines the hyperdynamic state of the myocardium, causing hypertrophy. In our study, we also found that patients with severe anemia were characterized by a high risk of LVH development. The influence of anemia on the process of calcification may be due to the action of hypoxia leading to increased CVC risk.

Soft-tissue calcification is a prominent feature in both chronic kidney disease (CKD) and experimental Klotho deficiency. Wild-type mice with CKD had very low renal, plasma, and urinary levels of Klotho [18]. In humans, Hu et al. observed a graded reduction in urinary Klotho starting at an early stage of CKD and progressing with loss of renal function [18]. The beneficial effect of Klotho on vascular calcification was a result of more than its effect on renal function and phosphatemia, suggesting a direct effect of Klotho on the vasculature. Our study also found a direct association between reduced serum alpha-Klotho level and high CRP and the relationship between two studied morphogenetic proteins and $\beta 2$-microglobulin. Based on these data it is possible to use serum FGF-23 and alpha-Klotho as diagnostic markers [19]. The results of the study allowed us to make the original predicting scheme for development of CVC, geometric remodeling, and functional alterations of $\mathrm{LV}$, all of which indicates that the process of calcification is not only a calciumphosphorus disorder.

M. Nasrallah defined that FGF-23 and ACI were significantly increased, and FGF-23 was independently associated with aortic calcification in haemodialysis patients [20]. Fibroblast growth factor 23 (FGF23) is the latest mineral metabolite to be linked to CVD and death. Like phosphate, PTH, and vitamin D before it, altered FGF23 levels were first recognized as an independent risk factor for mortality in dialysis patients [21] but later were shown to be a risk factor for CVD and death in the general population [22]. M. Kanbay et al. [23] provide the latest report on FGF23 and cardiovascular disease. In a cross-sectional study, the authors analyzed coronary angiograms from 177 patients who had "mild CKD" and for whom standard mineral metabolites were measured along with FGF23 and fetuin A levels. This study suggested that in a relatively young population with mild-to-moderate alteration of kidney function and with less traditional cardiovascular risk factors, anomalies of the serum FGF 23 and fetuin A levels appeared early in the course of disease and were independent major predictors for extent of coronary artery disease.

\section{Conclusion}

The study and identification of factors contributing to an increased risk for $\mathrm{CCV}$ is important for HD patients. The research into new $\mathrm{CCV}$ factors will help to improve the quality and length of life for those patients who receive RRT. 


\section{Competing interests}

The authors declare that they have no competing interests.

\section{References}

1. Moody WE, Edwards NC, Chue CD, Ferro CJ, Townend JN. Arterial disease in chronic kidney disease. Heart. 2013; 99(6):365-72.

2. Foley RN, Collins AJ, Herzog CA, Ishani A, Kalra PA. Serum phosphorus levels associate with coronary atherosclerosis in young adults. J Am Soc Nephrol. 2009;20: 397-404.

3. Block GA, Klassen PS, Lazarus JM, Ofsthun N, Lowrie EG, Chertow GM. Mineral metabolism, mortality, and morbidity in maintenance hemodialysis. J Am Soc Nephrol. 2004;15:2208-18.

4. Ganesh SK, Stack AG, Levin NW, Hulbert-Shearon T, Port FK. Association of elevated serum $\mathrm{PO}(4), \mathrm{Ca} \times \mathrm{PO}(4)$ product, and parathyroid hormone with cardiac mortality risk in chronic hemodialysis patients. J Am Soc Nephrol. 2001; 12:2131-8.

5. Forrest LM, McMillan DC, McArdle CS, Angerson WJ, Dagg K, Scott HR. A prospective longitudinal study of performance status, an inflammation-based score (GPS) and survival in patients. Br J Cancer. 2005;92(10):1834-6.

6. Kauppila LI, Polak JF, Cupples LA, Hannan MT, Kiel DP, Wilson PW. New indices to classify location, severity and progression of calcific lesions in the abdominal aorta: a 25year follow-up study. Atherosclerosis. 1997;132(2):245-50.

7. Rambod M, Bross R, Zitterkoph J, Benner D, Pithia J, Colman S, et al. Association of Malnutrition-Inflammation Score with quality of life and mortality in hemodialysis patients: a 5-year prospective cohort study. Am J Kidney Dis. 2009;53(2):298-309.

8. Fouque D, McKenzie J, de Mutsert R, Azar R, Teta D, Plauth M, et al. Use of a renal-specific oral supplement by haemodialysis patients with low protein intake does not increase the need for phosphate binders and may prevent a decline in nutritional status and quality of life. Nephrol Dial Transplant 2008; 8 (9):2902-10.

9. Stenvinkel P, Alvestrand A. Inflammation in end-stage renal disease: sources, consequences, and therapy. Semin Dial. 2002;15(5):329-37.

10. Rudenko LI, Batjushin MM, Kastanajan AA, Vorob'ev BI. Cardiovascular calcification risk prognosis in patients receiving chronic hemodialysis. Nefrologiia. 2015;19(5):726. [Article in Russian].

11. Yan L, Mathew L, Chellan B, Gardner B, Earley J, Puri TS, et al. S100/Calgranulin-mediated inflammation accelerates left ventricular hypertrophy and aortic valve sclerosis in chronic kidney disease in a receptor for advanced glycation end products-dependent manner. Arterioscler Thromb Vasc Biol. 2014;34(7):1399-411.

12. Barry SP, Townsend PA. What causes a broken heart-molecular insights into heart failure. Int Rev Cell Mol Biol 2010; 284:113-79.

13. Lee JK, Lin HH, Tsai CT, Chen JJ, Kuo CC, Lien YC, et al. Differential association of proinflammatory cytokines with left ventricular diastolic dysfunction in subjects with and without continuous ambulatory peritoneal dialysis. Nutr Metab Cardiovasc Dis. 2012;22(11):974-80.

14. Smeets PJ, Teunissen BE, Planavila A, de Vogelvan den Bosch H, Willemsen PH, van der Vusse GJ, et al. Inflammatory pathways are activated during cardiomyocyte hypertrophy and attenuated by peroxisome proliferatoractivated receptors PPARalpha and PPARdelta. J Biol Chem. 2008; 283(43):29109-18.

15. Wang AY, Wang M, Woo J, Lam CW, Lui SF, Li PK, et al. Inflammation, residual kidney function, and cardiac hypertrophy are interrelated and combine adversely to enhance mortality and cardiovascular death risk of peritoneal dialysis patients. J Am Soc Nephrol. 2004;15(8);2186-94.

16. Masuda M, Ishimura E, Ochi A, Tsujimoto $Y$, Tahahra H, Okuno S, et al. Serum $\beta 2$-microglobulin correlates positively with left ventricular hypertrophy in long-term hemodialysis patients. Nephron Clin Pract. 2014;128(1-2):101-6.

17. Aronow WS, Ahn C, Kronzon I. Association of mitral annular calcium with symptomatic peripheral arterial disease in older persons. Am J Cardiol. 2001;88(3):333-4.

18. Hu MC, Shi M, Zhang J, Quiñones H, Griffith C, Kuro-o $\mathrm{M}$, et al. Klotho deficiency causes vascular calcification in chronic kidney disease. J Am Soc Nephrol. 2011;22(1):124-36. 19. Rudenko LI, Batjushin MM, Kastanajan AA, Vorob'ev BI, Sarvilina IV. The serum alpha-Klotho, FGF-23 and their participation in cardiovascular calcification. Klinicheskaja nefrologija. 2015;1:23-6. [ Article in Russian].

20. Nasrallah MM, El-Shehaby AR, Salem MM, Osman NA, El Sheikh E, Sharaf El, et al. Fibroblast growth factor-23 (FGF-23) is independently correlated to aortic calcification in haemodialysis patients. Nephrol Dial Transplant. 2010;25(8): 2679-85.

21. Gutierrez OM, Mannstadt M, Isakova T, Rauh-Hain JA, Tamez H, Shah A, et al. Fibroblast growth factor 23 and mortality among patients undergoing hemodialysis. N Engl J Med. 2008;359:584-92.

22. Ix JH, Shlipak MG, Wassel CL, Whooley MA. Fibroblast growth factor-23 and early decrements in kidney function: The Heart and Soul Study. Nephrol Dial Transplant. 2010; 25:993-7.

23. Kanbay M, Nicoleta M, Selcoki Y, Ikizek M, Aydin M, Eryonucu B, et al. Fibroblast growth factor 23 and fetuin A are independent predictors for the coronary artery disease extent in mild chronic kidney disease. Clin J Am Soc Nephrol. 2010;5:1780-86. 